\title{
Magnetic field induced orientational bistability in a ferronematic cell
}

\author{
S.V. BURYLOV ${ }^{1}$, V.I. ZADOROZHNII ${ }^{2}$, I.P. PINKEVICH ${ }^{2}$, \\ V.YU. RESHETNYAK ${ }^{2}$ AND T.J. SLUCKIN ${ }^{3}$ \\ ${ }^{1}$ Institute of Transport Systems and Technologies, Nat. Acad. of Sci. \\ of Ukraine, Pisargevskogo St., 5, Dnepropetrovsk, 49005, Ukraine; \\ ${ }^{2}$ Kyiv National Taras Shevchenko University, Physics Faculty, Prosp. \\ Glushkova, 6, Kyiv, 03022, Ukraine; \\ ${ }^{3}$ University of Southampton, Faculty of Mathematical Studies \\ Southampton, SO17 1BJ, United Kingdom
}

The equilibrium states of a suspension of single-domain ferroparticles in a nematic liquid-crystalline homeotropic cell subject to an external magnetic field are studied. We predict the existence of magnetic field induced orientational bistability in such a system in a magnetic field $\sim 10^{2}$ Oe. The existence of the bistability phenomenon is governed by conditions on the cell thickness and on the director anchoring energy. The effect can be controlled using a small bias magnetic field normal to the unperturbed director. The director reorientation in a magnetic field causes an effective change in refraction index, which enables the orientational bistability to be exploited in optical devices.

Keywords: liquid crystal; ferroparticle; magnetic field; bistability 


\section{S.V. BURYLOV et al.}

\section{INTRODUCTION}

In the last ten years there has been increasing interest in liquid crystals (LC) containing magnetic particles. These systems are both of generalphysical interest and have considerable potential for use in technical, in part optical, applications ${ }^{[1-4]}$. The key property of a nematic crystal doped with single-domain ferromagnetic particles, known as a ferronematic $(\mathrm{FN})$, is the inherent high magnetic susceptibility making it possible to exert control of its orientational texture using weak $(<10$ Oersteds) magnetic fields.

Magnetic suspensions in nematic liquid crystals were proposed and considered by Brochard and de Gennes ${ }^{[1]}$. Much later thermotropic FN's were synthesised by Chen and Amer ${ }^{[5]}$ using a MBBA LC matrix. The initial continuum theory ${ }^{[1]}$ was generalized by Burylov and Raikher ${ }^{[6]}$ to include a finite nematic anchoring energy at the ferroparticle surface. This provided an explanation for the absence of $\mathrm{co}^{-}$ alignment of the nematic director and averaged local magnetization in the FN, as was found experimentally (see, for example, Ref. ${ }^{[7]}$ ). The generalized theory has been confirmed in experiments, among them one on 8CB-based FN with almost spherical magnetite particles ${ }^{[2]}$.

Since these early studies, peculiarities have been noted in behavior of field dependencies of birefringence in ferronematics containing needle-like magnetic particles. The phase retardation between the extraordinary and ordinary rays exhibits saturation or nonmonotonic behavior in the fields $\geq 10$ Oe, depending upon cell thickness and the concentration of ferroparticles. There are phase retardation oscillations caused by ferroparticle coagulation as well as a monotonic increase in the phase retardation followed by a decrease for fields $\geq 40$ Oe. These were confirmed by numerical simulations reported in Ref. ${ }^{[6]}$. In this paper, we demonstrate the consequences of this feature for a homeo- 


\section{MAGNETIC FIELD INDUCED ORIENTATIONAL . . .}

tropic FN cell. Specifically, we predict the existence of a new phenomenon: magnetic field induced orientational bistability in nematic liquid crystals containing monodomain ferromagnetic particles.

\section{FORMULATION OF THE PROBLEM}

Let us consider a cell of thickness $D$ filled with FN. We suppose homeotropic nematic anchoring of finite strength $W_{0}$ and $W_{D}$ at the lower $(z=0)$ and upper $(z=D)$ cell planes, respectively. We also suppose an anchoring energy of strength $W_{P}$ at the ferroparticle surfaces favoring soft homeotropic anchoring. The ferroparticles will be modelled as needle-like monodomain ferrite grains of length $L$ and diameter $d \approx L / 7$ significantly greater than the nematic molecule size. The cell is initially subjected to a small bias magnetic field $H_{b}$ parallel to the cell planes. In this initial state ferroparticles are homogeneously distributed over the cell volume and their magnetic moments are perpendicular to the unperturbed director $\mathbf{n}_{0}$. The sample is magnetized up to saturation, which is attained for the ratio of magnetic field energy to heat energy $\rho_{b}=M_{s} \nu H_{b} / k_{B} T \geq 10^{[6]}$. Here $M_{s}$ is the saturation magnetization of the particle substance, $\nu$ is the volume of the particle. For $\gamma-\mathrm{Fe}_{2} \mathrm{O}_{3}$ particles $M_{s} \sim 340 \mathrm{G}, \nu \sim 2 \times 10^{-15} \mathrm{~cm}^{3}$ and we have $\rho_{b} \geq 10$ at $H_{b} \geq 0.6$ Oe and $T=25^{\circ} \mathrm{C}$.

To observe the orientational bistability phenomenon, an external variable magnetic field $H$ of moderate strength $(\sim 50-150$ Oe $)$ should be applied to the cell normally to its planes. The orientational texture of FN and the choice of the reference frame are shown in Figure 1. A magnetic field $H$ rotates the ferroparticles producing distortions within the LC matrix. The volume fraction $f$ of the particles, at given thickness of the cell, will be considered to exceed the critical value $f^{*} \sim 2 K \nu /\left(\pi d L W_{P} D^{2}\right)$, where $K$ is the mean value of the nematic 


\section{S.V. BURYLOV et al.}

elastic constants, yielding the collective behavior of the particles ${ }^{[1,6]}$. Induced distortions of the particles in this case are imparted to the LC matrix bringing about macroscopic changes in the sample texture.
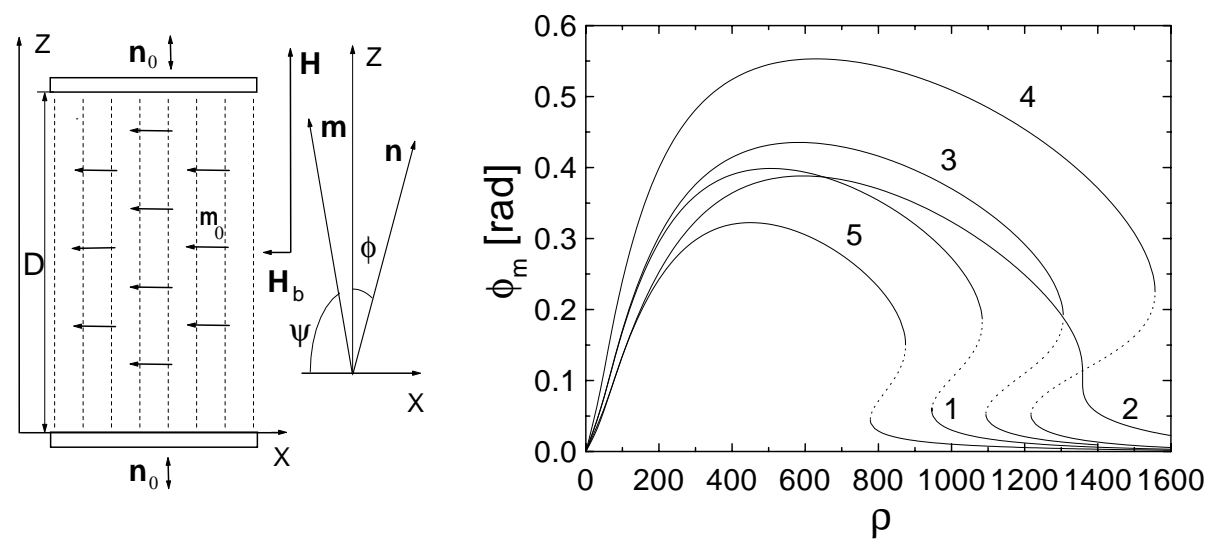

FIGURE 1 Schematic FIGURE 2 The angle $\phi_{m}$ vs $\rho$ for $p=0$ of the FN cell.

(1) $D / \lambda=0.1, \quad w_{0}=w_{D}=3, \quad \sigma=200$, $\rho_{b}=10,(2)$ the same values, but $\rho_{b}=68$. (3) $D / \lambda=0.1, \quad w_{0}=w_{D}=3, \quad \sigma=220$, (4) $D / \lambda=0.12, w_{0}=w_{D}=3, \sigma=200$, (5) $D / \lambda=0.1, w_{0}=1000, w_{D}=3, \sigma=$ $200 ; \rho_{b}=10$.

The FN equilibrium state at given $\mathbf{H}_{p}=\mathbf{H}+\mathbf{H}_{b}$ can be determined by minimizing the full free energy functional ${ }^{[6]}$ :

$$
\begin{aligned}
F & =\int_{V}\left\{\frac{1}{2}\left[K_{1}(\operatorname{div} \mathbf{n})^{2}+K_{2}(\mathbf{n} \cdot \operatorname{curl} \mathbf{n})^{2}+K_{3}(\mathbf{n} \times \operatorname{curl} \mathbf{n})^{2}\right]\right. \\
& \left.-\frac{1}{2} \chi_{a}\left(\mathbf{n} \cdot \mathbf{H}_{p}\right)^{2}+\frac{f k_{B} T}{\nu} \ln f-M_{s} f\left(\mathbf{m} \cdot \mathbf{H}_{p}\right)+\frac{f W_{p}}{d}(\mathbf{n} \cdot \mathbf{m})^{2}\right\} d V \\
& +\int_{S_{0}} \frac{W_{0}}{2}\left(\mathbf{n} \times \mathbf{n}_{0}\right)^{2} d S+\int_{S_{D}} \frac{W_{D}}{2}\left(\mathbf{n} \times \mathbf{n}_{0}\right)^{2} d S
\end{aligned}
$$




\section{MAGNETIC FIELD INDUCED ORIENTATIONAL . . .}

in combination with condition $\int_{V} f d V=\bar{f} V$, where $\mathbf{m}$ is the unit vector in the direction of the sample magnetization, $K_{j}$ are elastic constants, $\chi_{a}$ is the anisotropic part of the diamagnetic susceptibility of nematic and $\bar{f}$ is the mean particle volume fraction. The volume integral in Eq. (1) describes the FN bulk free energy in a magnetic field ${ }^{[6]}$. The last two terms are the nematic surface potentials.

In this geometry, the director $n$ and the unit vector $\mathbf{m}$ are given by $\mathbf{n}=[\sin \phi, 0, \cos \phi], \mathbf{m}=[-\cos \psi, 0, \sin \psi]$, where $\phi=\phi(z)$ and $\psi=\psi(z)$. In what follows we use the parameters intrinsic to this system: $\lambda=\left(K_{3} \nu / 2 \bar{f} k_{B} T\right)^{1 / 2}$ (the FN magnetic coherence length ${ }^{[6]}$ ) and dimensionless parameters: $\sigma=\nu W_{P} / d k_{B} T, \rho=M_{s} \nu H / k_{B} T, w_{0}=$ $W_{0} D / K_{3}, w_{D}=W_{D} D / K_{3}, p=K_{1} / K_{3}-1$, and $\kappa=\chi_{a} k_{B} T / 2 \bar{f} \nu M_{s}^{2}$. Minimization of the functional (1) implies a set of equations for equilibrium profiles $\phi(z), \psi(z), f(z)$ and boundary conditions on the cell planes (see details in Ref. ${ }^{[6]}$ ). These equations were solved numerically.

\section{RESULTS OF SIMULATIONS AND DISCUSSION}

The distortions within the LC matrix increase initially with the magnetic field strength $H$. When $\rho \geq(1.5-2) \sigma$, two types of orientational deformations can occur, depending on cell thickness ${ }^{[8]}$. For $D>D_{c r}=\left(\pi \lambda / \sigma^{1 / 2}\right) C$, as $\rho$ increases ferroparticles tend to align parallel to $\mathbf{H}$, forming a high particle concentration zone inside the cell. Here $C$ is a factor whose analytical expression is known only for very large and very small values of $w_{0}$ and $w_{D}$ as compared to unity ${ }^{[8]}$. Note that $D_{c r}=D^{*} \pi C$, where $D^{*}=\lambda / \sigma^{1 / 2}$ is the critical value of the cell thickness ${ }^{[8]}$, above which the collective behavior of the particles takes place. When $D^{*}<D<D_{c r}$, the nematic director is decoupled from the ferroparticles with increasing $\rho$ and the concentration tends to 


\section{S.V. BURYLOV et al.}

be homogeneous. In the transitional region between small and strong decoupling the FN director possesses two steady-state spatial orientations in some range of the magnetic field strengths.

We have performed numerical simulations for cells of thickness $D^{*}<D<D_{c r}$ such that the nematic director is decoupled from ferroparticles in strong enough magnetic field and orientational bistability can occur. As an illustration we have considered a ferronematic with rod-like magnetite particles with $d \sim 70 \mathrm{~nm}$ and $L \sim 500 \mathrm{~nm}$ $\left(\rho / H \approx 16 \mathrm{Oe}^{-1}\right.$ at $\left.25^{0} \mathrm{C}\right) . H_{b}$ should be $\geq 0.6$ Oe $\left(\rho_{b} \geq 10\right)$ so as to magnetize FN nearly perfectly. For chosen value of $\sigma=200, W_{P}$ is $3 \times 10^{-2} \mathrm{erg} \mathrm{cm}^{-2}$, which is compatible with known values for real systems (see, for example, Ref. [6]). To ensure the collective behavior of the ferroparticles, the thickness of the cell must exceed the critical value $D^{*}$. It follows that the ratio $D / \lambda$ must be greater than $\sigma^{-1 / 2}$. This imposes the restriction that $D^{2} f>K_{3} \nu / 2 \sigma k_{B} T$. For MBBAbased FN $K_{3} \approx 7.5 \times 10^{-7}$ dyn and we have $D^{2} f>8.8 \times 10^{-11} \mathrm{~cm}^{2}$. As a consequence, for $D=340 \mu m$ the mean volume fraction of ferroparticles $\bar{f}=\bar{c} \nu$ must be greater than $7.6 \times 10^{-8}$ ( $\bar{c}$ is the mean number concentration). In our calculations we put $\bar{f}=1.5 \times 10^{-7}$ and $\chi_{a}=0.97 \times 10^{-7}$.

The set of equilibrium equations was solved numerically for the actual ferronematic system. The solutions obtained were tested for stability with respect to perturbations. A numerical investigation showed that the solutions corresponding to the middle part of the hysteresis loop are unstable.

Figure 2 shows the behavior of the hysteresis loop for different values of parameters of the system. The hysteresis loop shifts towards larger values of $\rho$ and the loop area enlarges as $\sigma$ and/or $D / \lambda$ increase (curves $1,3,4$ ). Curves 1 and 5 illustrate the effect of weak and strong 


\section{MAGNETIC FIELD INDUCED ORIENTATIONAL ...}

anchoring at the lower orienting surface of the cell. An increase in $H_{b}$ leads to a reduction in the loop area and the disappearance of the hysteresis at specific $H_{b}=H_{b}^{*}$ (curves 1 and 2). This permits the phenomenon to be controlled using small bias magnetic field $(\sim 4.25$ Oe in Figure 2).

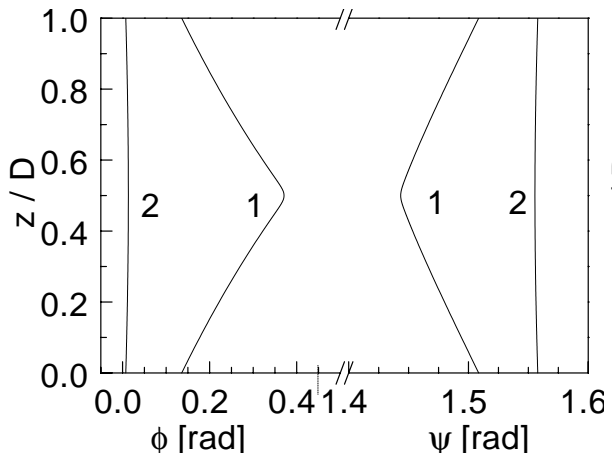

(a)

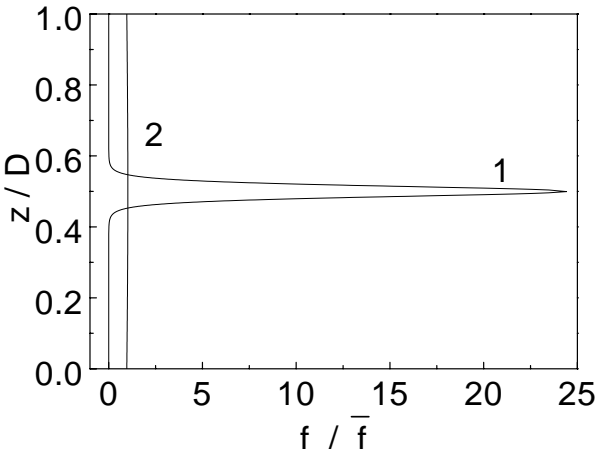

(b)

FIGURE 3 Calculated (a) orientational and (b) concentration profiles at $D / \lambda=0.12, w_{0}=w_{D}=3, \sigma=200, \rho_{b}=10$, $p=0$ and $\rho=1400$. Curves 1 and 2 correspond to the upper and lower parts of the hysteresis loop.

Orientation and concentration profiles inside the FN cell are shown in Figures 3 (a) and 3 (b), respectively, for orientational bistability region. In strong enough magnetic field under condition $D<D_{c r}$ both the nematic director and the magnetic moment of each particle are aligned normally to the planes of the cell and parallel to $\mathbf{H}$ (strong nematic director decoupling; curves 2 in Figure 3 (a)). In this case, the concentration of ferroparticles is uniform within the cell (curve 2 in Figure $3(\mathrm{~b})$ ). As magnetic field decreases, the contribution of the particle-to-director anchoring energy increases. The torque exerted on the nematic by the particle rises, restoring the initial homeotropic 


\section{S.V. BURYLOV et al.}

orientation of a ferroparticle in a nematic matrix. An increase in the angle $\beta$ between local $\mathbf{m}(z)$ and $\mathbf{n}(z)$ results in distortion of the LC matrix. Once the turning point in the hysteresis loop is reached, the system turns into a new stable state and the LC matrix distortion rises sharply (see Figure 3 (a)); ferroparticles transfer inward from the planes of the cell minimizing their orientational energy. Consequently, the ferroparticles gather within a narrow region inside the cell (the segregation effect ${ }^{[1,6]}$; see Figure $3(b)$ ). The degree of the FN layer compression is controlled by entropy springiness of the ferroparticle solution.

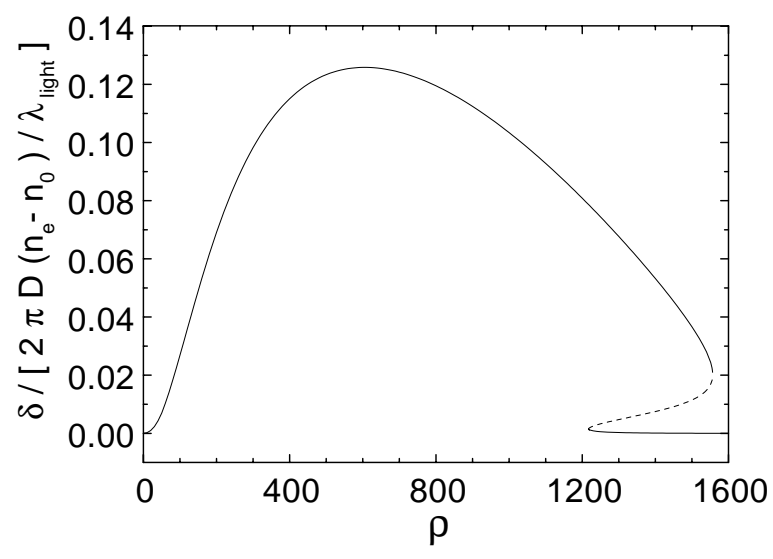

FIGURE 4 Phase retardation vs normalized magnetic field at $\lambda_{\text {light }}=$ $514.5 \mathrm{~nm}, D / \lambda=0.12, w_{0}=w_{D}=3, \sigma=200, \rho_{b}=10$ and $p=0$.

With increasing magnetic field, the energy goes into rotating the ferroparticles and the consequent decoupling of the nematic director. A decrease in stored elastic energy and magnetic energy of the LC matrix is accompanied by a decrease in the angle $\phi$. With decoupling of the nematic director the angle $\beta$ decreases and the FN stratification decreases. A predominance of the expenditure of energy into rotating 


\section{MAGNETIC FIELD INDUCED ORIENTATIONAL . . .}

the ferroparticles over decoupling of the nematic director terminates in sharp decoupling of the director. An increase in $H_{b}$ causes the braking torque to rise. This involves a redistribution of the contributed energies and, as noted above, starting with some $H_{b}^{c r}$ sharp decoupling changes to smooth one (see Figure 2). In the latter case, there is no hysteresis.

A flat layer of $\mathrm{FN}$ is birefringent for light beam propagating along the z-axis. The phase retardation between the extraordinary and ordinary rays depends on the distortion of the LC matrix and exhibits bistable behavior (see Figure 4).

\section{CONCLUSIONS}

In conclusion, we have extended previous studies on macroscopic properties of ferronematics in external magnetic field caused by orientational interactions on the ferroparticles surfaces ${ }^{[8]}$ to the demonstration of new possible effects in a moderate magnetic field. Specifically, we predict the existence of orientational hysteresis in a homeotropic ferronematic cell governed by conditions on the cell thickness and on the director anchoring energy at the ferroparticle surface and planes of the cell. A direct consequence of this effect is magnetic field induced orientational bistability in such a system. The phenomenon under consideration can occur if the thickness of the cell is in the range from $D^{*}$ to $D_{c r}=D^{*} \pi C$, where $D^{*}=\left(K_{3} d / 2 \bar{f} W_{P}\right)^{1 / 2}$, and the magnetic field

$H>H_{c}>\sqrt{2} W_{P} / M_{s} d+H_{b}$. The transition between strong and slightly distorted states of the LC matrix is sharp and is accompanied by sharp change in optical phase retardation of a FN layer. Small bias magnetic fields $(<10 \mathrm{Oe})$, parallel to the planes of the cell, influence the width of the hysteresis loop, thereby enabling the effect to be controlled. Reasonably strong bias magnetic fields suppress the hysteresis. This effect can be used under optimized conditions in bistable devices, 


\section{S.V. BURYLOV et al.}

in polarization analysis, and also for investigating the properties of liquid crystals.

\section{Acknowledgments}

This work has been partially supported by INTAS grant N 99-00312.

\section{References}

[1] F. Brochard and P.G. de Gennes, J. Phys. (France), 31, 691 (1970).

[2] Potočová, M. Koneracká, P. Kopčanský, M. Timko, L. Tomčo, J. Jadzyn and G. Czechowski, JMMM, 201, 163 (1999).

[3] Yu.L. Raikher and V.I. Stepanov, JMMM, 201, 182 (1999).

[4] V. Berejnov, J.-C. Bacri, V. Cabuil, R. Perzynski and Yu. Raikher, Europhys. Lett. 41, 507 (1998).

[5] S.-H. Chen and N.M. Amer, Phys. Rev. Lett. 51, 2298 (1983).

[6] S.V. Burylov and Yu.L. Raikher, Mol. Cryst. Liq. Cryst. 258, 107; ibid 258, 123 (1995).

[7] B.J. Liang and S.-H. Chen, Phys. Rev. A, 39, 1441 (1989).

[8] S.V. Burylov, V.I. Zadorozhnii, I.P. Pinkevich, V.Yu. Reshetnyak and T.J. Sluckin, JMMM (in press). 